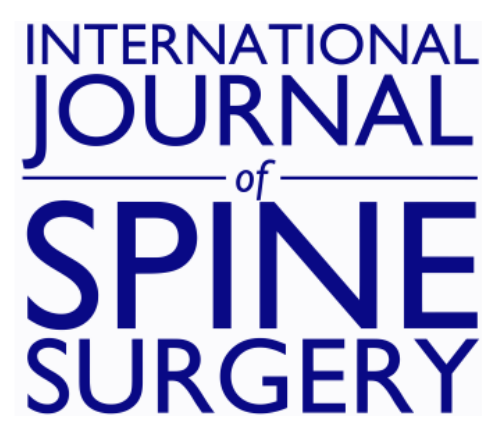

\title{
Long-Term Clinical Experience with Selectively Constrained SECURE-C Cervical Artificial Disc for 1-Level Cervical Disc Disease: Results from Seven-Year Follow-Up of a Prospective, Randomized, Controlled Investigational Device Exemption Clinical Trial
}

\author{
ALEXANDER VACCARO, WILLIAM BEUTLER, WALTER PEPPELMAN, JOSEPH \\ MARZLUFF, ANDREW MUGGLIN, PREM S. RAMAKRISHNAN, JACQUELINE MYER and \\ KELLY J. BAKER
}

Int J Spine Surg 2018, 12 (3) 377-387

doi: https://doi.org/10.14444/5044

http://ijssurgery.com/content/12/3/377

This information is current as of April 26, 2023.

Email Alerts Receive free email-alerts when new articles cite this article. Sign up at:

http://ijssurgery.com/alerts 


\title{
Long-Term Clinical Experience with Selectively Constrained SECURE-C Cervical Artificial Disc for 1-Level Cervical Disc Disease: Results from Seven-Year Follow-Up of a Prospective, Randomized, Controlled Investigational Device Exemption Clinical Trial
}

\author{
ALEXANDER VACCARO, MD, PHD,${ }^{1}$ WILLIAM BEUTLER, $\mathrm{MD},{ }^{2}$ WALTER PEPPELMAN, DO,${ }^{2}$ JOSEPH \\ MARZLUFF, MD ${ }^{3}$ ANDREW MUGGLIN, PHD, ${ }^{4}$ PREM S. RAMAKRISHNAN, PHD,${ }^{5}$ JACQUELINE \\ MYER, ${ }^{5}$ KELLY J. BAKER, PHD ${ }^{5}$ \\ ${ }^{1}$ Rothman Institute, Philadelphia, Pennsylvania, ${ }^{2}$ Pennsylvania Spine Institute, Harrisburg, Pennsylvania, ${ }^{3}$ Trident Regional Medical Center, Charleston, South \\ Carolina, ${ }^{4}$ Paradigm Biostatistics LLC, Anoka, Minnesota, ${ }^{5}$ Globus Medical, Audubon, Pennsylvania
}

\begin{abstract}
Background: This research was initiated to compare the long-term clinical safety and effectiveness of the selectively constrained SECURE-C (Globus Medical, Audubon, Pennsylvania) Cervical Artificial Disc to anterior cervical discectomy and fusion (ACDF). To preserve segmental motion, cervical total disc replacement (CTDR) was developed as an alternative to ACDF. Current CTDR designs incorporate constrained and unconstrained metal-onmetal or metal-on-polymer articulation with various means of fixation.

Methods: Eighteen investigational sites participated in this prospective clinical trial; 380 patients were enrolled and treated in the investigational device exemption study. The first 5 patients treated at each site were nonrandomized and received the investigational SECURE-C device. Patients were randomized, treated surgically, and evaluated postoperatively at 6 weeks, 3 months, 6 months, 12 months, 24 months, and annually thereafter through 84 months postoperative.

Results: Overall results for the randomized cohorts demonstrated statistical superiority of the investigational SECURE-C group over the control ACDF group at 84 months postoperative. SECURE-C showed clinically significant improvement in pain and function in terms of neck disability index and visual analog scale scores, and superiority in patient satisfaction was also achieved for patients treated with SECURE-C.

Conclusion: Clinical study results indicated that the selectively constrained SECURE-C Cervical Artificial Disc is as safe and effective as ACDF. Long-term results from the Post Approval Study demonstrated that SECURE-C is statistically superior to ACDF in terms of overall success and patient satisfaction. Lower rates of subsequent index-level surgeries and device-related adverse events were observed in the SECURE-C group than in the ACDF group. The long-term, level 1 clinical evidence presented here is consistent with other reports supporting the safety and efficacy of cervical arthroplasty, and furthers advocacy for motion preservation as a viable alternative to fusion.
\end{abstract}

Cervical Spine

Keywords: cervical, total disc arthroplasty, motion preservation, total disc replacement, anterior cervical discectomy and fusion, follow-up, 84 months, noninferiority, superiority, selectively constrained, adverse events, clinical trial, symptomatic, cervical disc disease, Bayesian

\section{INTRODUCTION}

Surgical treatment of symptomatic cervical disc disease (CDD) is achieved by neural decompression and, if required, stabilization of the treated level. Fusion after discectomy is currently the standard of care employed for various pathological conditions of the spine. In the cervical spine, anterior cervical discectomy and fusion (ACDF) is a successful procedure and is reported to provide excellent symptom relief. However, there is reasonable concern about the long-term effects of fusion on the overall health of the cervical spine, especially with current trends showing an increased number of cervical fusions in the United States and abroad during the past 3 decades. ${ }^{1-3}$

As an alternative to fusion, artificial discs preserve motion at the treated level while providing 
commensurate symptom relief. In addition to mitigating complications attributed to fusion procedures, it has been postulated that motion preservation devices may delay or even prevent the onset of symptomatic adjacent-level disease. ${ }^{4,5}$

This study reports the 7-year safety and effectiveness data of the SECURE-C (Globus Medical, Audubon, Pennsylvania) Cervical Artificial Disc, a selectively constrained cervical total disc replacement (CTDR) device. The design of SECURE-C selectively constrains motion through controlled articulation to more closely replicate the cervical spine's natural motion. This study is a prospective, multicenter, 2-arm, randomized (1:1), concurrently controlled study treating patients with intractable symptomatic CDD at a single level between $\mathrm{C} 3$ and C7 with CDTR being compared to traditional ACDF.

\section{MATERIALS AND METHODS}

\section{Clinical Study Design}

A multicenter, prospective, randomized investigational device exemption (IDE) clinical study was conducted to compare the safety and effectiveness of the SECURE-C Cervical Artificial Disc to control ACDF. A total of 380 patients were enrolled from July 2005 to April 2008 at 18 sites, according to inclusion/exclusion criteria defined in the Food and Drug Administration (FDA)-approved clinical protocol. The first 5 subjects enrolled at each center were nonrandomized and received the SECURE-C device ( 89 patients). Of the 291 randomized patients, 151 were randomized to SECURE-C and 140 to control ACDF treatment. The most commonly implanted SECURE-C device was the 13-mm (AP depth) $\times 14-\mathrm{mm}($ ML width) in 7-mm-height device. While the device was available in 2 sagittal $\left(0^{\circ}\right.$ and $6^{\circ}$ lordosis) profiles, the $6^{\circ}$ lordosis option was most commonly used. The FDA granted premarket approval of the SECURE-C Cervical Artificial Disc after evaluating the 24-month results submitted for the IDE study. Study design, inclusion/exclusion criteria, and significant clinical findings at the 24month window were previously reported. ${ }^{6}$

Subsequent to premarket approval, subjects treated in the IDE study were to be followed in a related postmarket approval study (PAS). The PAS was designed to include all subjects who returned for 24-month follow-up (334 subjects). However, sites were requested to gain the consent of all IDE patients who were willing to return for follow-up at later time points. Patients who had secondary surgery related to the index level were considered to be cumulative failures. Results presented here are from the 7-year follow-up in the PAS.

\section{Clinical Outcome Measures}

\section{Primary Endpoint: Overall Study Success}

Overall success (referred to as protocol-specified) was defined as a composite score whereby a patient was considered a success if all of the following conditions were met:

1. Pain/disability improvement of at least $25 \%$ in neck disability index (NDI) compared with baseline

2. No device failures requiring revision, removal, reoperation, or supplemental fixation

3. Absence of major complications defined as major vessel injury, neurological damage, or nerve injury

4. For patients who underwent ACDF only, radiographic fusion, as defined by the presence of bridging trabecular bone, without evidence of pseudarthrosis (defined radiographically as no apparent bridging trabecular bone and range of motion $(\mathrm{ROM})>3 \mathrm{~mm}$ in translation and $>2^{\circ}$ in rotation)

Additionally, more rigorous FDA-specified criteria (FDA-defined) for overall success was defined as outlined below:

1. Pain/disability improvement of at least 15 points in NDI compared with the baseline

2. No secondary surgery at the index level, including revision, removal, reoperation, or supplemental fixation

3. No potentially device-related adverse events

4. Maintenance or improvement in all components of neurological status

5. No SECURE-C intraoperative changes in treatment

\section{Secondary Endpoints}

The secondary endpoint evaluations included components of the primary endpoint (NDI improvement; no device failure requiring revision, reoperation, or removal; absence of major complications), NDI success $(\geq 25 \%$ and $\geq 15$-point improvement from baseline), visual analog scale (VAS) neck pain, left arm pain, and right arm pain success $(\geq 20-\mathrm{mm}$ 
Table 1. Overall success (protocol-specified) for all groups.

\begin{tabular}{|c|c|c|c|c|c|c|}
\hline \multirow[b]{2}{*}{ Component } & \multirow{2}{*}{$\begin{array}{c}\text { Nonrandomized } \\
\text { SECURE-C } \\
(\mathbf{N}=89)\end{array}$} & \multirow{2}{*}{$\begin{array}{c}\text { Randomized } \\
\text { SECURE-C } \\
(\mathrm{N}=151)\end{array}$} & \multirow{2}{*}{$\begin{array}{c}\text { Randomized } \\
\text { ACDF } \\
(\mathbf{N}=140)\end{array}$} & \multicolumn{2}{|c|}{ Posterior Probability } & \multirow{2}{*}{$\begin{array}{c}95 \% \text { BCI* } \\
\text { (Lower, Upper) }\end{array}$} \\
\hline & & & & Noninferiority & Superiority & \\
\hline NDI ( $\geq 25 \%$ improvement) & $53 / 63(84.1 \%)$ & $113 / 125(90.4 \%)$ & $92 / 107(86.0 \%)$ & $100.0 \%$ & $86.8 \%$ & $(-3.6 \%, 13.3 \%)$ \\
\hline No removals, etc. & $61 / 65(93.8 \%)$ & $124 / 131(94.7 \%)$ & $102 / 121(84.3 \%)$ & $100.0 \%$ & $99.6 \%$ & $(2.4 \%, 16.9 \%)$ \\
\hline No major complications & $63 / 63(100.0 \%)$ & $125 / 125(100.0 \%)$ & $107 / 107(100.0 \%)$ & $100.0 \%$ & $53.9 \%$ & $(-2.2 \%, 2.7 \%)$ \\
\hline Fusion (control) & N/A & $\mathrm{N} / \mathrm{A}$ & $101 / 104(97.1 \%)$ & $\mathrm{N} / \mathrm{A}$ & $\mathrm{N} / \mathrm{A}$ & $\mathrm{N} / \mathrm{A}$ \\
\hline Overall success & $53 / 65(81.5 \%)$ & $113 / 131(86.3 \%)$ & $84 / 120(70.0 \%)$ & $100.0 \%$ & $99.9 \%$ & $(6.3 \%, 25.8 \%)$ \\
\hline
\end{tabular}

Abbreviations: ACDF, anterior cervical discectomy and fusion; BCI, Bayesian credible intervals; NDI, neck disability index.

$*$ BCI for difference in proportions (SECURE-C - ACDF).

improvement from baseline or 0 -mm postoperative score), health status success using the 36-Item Short Form Health Survey (SF-36) for mental and physical composite scores (15\% improvement from baseline), and patient satisfaction success (definitely or mostly satisfied). Additionally, maintenance or improvement in neurological status (percentage maintained or improved in comparison to the preoperative baseline) was analyzed. Radiological findings including mean ROM (angulation and translation), change in disc height from baseline ( $>2-\mathrm{mm}$ changes), presence of radiolucency, formation of heterotopic ossification (HO) in the SECURE-C group, achievement of spinal fusion (control group only), and device displacement or migration $(>3 \mathrm{~mm})$ were also evaluated. All patient data were collected and reported to the study sponsor.

\section{Statistical Methods}

Bayesian statistical methods were prespecified for the analysis of primary and secondary objectives. Prior distributions for all parameters of interest were noninformative. The statistical plan stipulated a multiple imputation approach that predicted 84month outcomes for subjects without them, based on interim outcomes observed at 6 months and annually thereafter. Differences in proportions were tested for noninferiority; if noninferior, a test of superiority followed. For tests of proportions, a noninferiority margin (delta) of 0.10 was specified.

Concluding noninferiority required a posterior probability exceeding 0.95 , analogous to statistical significance at the (one-sided) $5 \%$ level in a traditional hypothesis test. The same threshold was used when testing superiority. In addition, equal-tailed 95\% Bayesian credible intervals (BCIs) were calculated for selected quantities; a 95\% BCI that excludes 0 indicates a statistical difference between quantities, analogous to statistical significance at the (2-sided) 5\% level for a traditional hypothesis test.

\section{Device Design}

The SECURE-C Cervical Artificial Disc is a selectively constrained anterior articulating intervertebral device comprised of a central core and 2 endplates. Details of its device design characteristics and function were previously described in the 24month follow-up. ${ }^{6}$

\section{RESULTS \\ Patient Follow-Up Rates}

Among the 380 patients enrolled in the IDE study, there were 4 deaths and 30 failures (including revisions, removals, supplemental fixation, and reoperations at the index level for the randomized cohorts) by 84 months postoperative, bringing the total number of patients eligible for long-term follow-up to 346 . The overall follow-up rate achieved at 84 months for patients with efficacy components was $82.7 \%(286 / 346)$ with individual follow-up rates of $81.9 \%(185 / 226)$ for the entire SECURE-C cohort (nonrandomized and randomized), $86.1 \%(124 / 144)$ for the randomized SECURE-C group, and $84.2 \%(101 / 120)$ for the ACDF group.

\section{Patient Demographics}

There were no differences with respect to gender, age, race (Caucasian vs. other), height, weight, body mass index, tobacco use, duration of symptoms, history and type of nonoperative care, or history and type of previous surgery between the 2 randomized groups as reported previously. ${ }^{6}$

\section{Clinical and Patient-Reported Outcomes}

\section{Overall Success}

The success rates at 84 months postoperative for overall success and each of the individual success components are provided in Tables 1 and 2. The individual patient success rate was defined as the 
Table 2. Overall success (FDA-defined) for all groups.

\begin{tabular}{|c|c|c|c|c|c|c|}
\hline \multirow[b]{2}{*}{ Component } & \multirow{2}{*}{$\begin{array}{l}\text { Nonrandomized } \\
\text { SECURE-C } \\
(\mathrm{N}=89)\end{array}$} & \multirow{2}{*}{$\begin{array}{c}\text { Randomized } \\
\text { SECURE-C } \\
(\mathrm{N}=151)\end{array}$} & \multirow{2}{*}{$\begin{array}{c}\text { Randomized } \\
\text { ACDF } \\
(\mathbf{N}=140) \\
\end{array}$} & \multicolumn{2}{|c|}{ Posterior Probability } & \multirow{2}{*}{$\begin{array}{c}95 \% \text { BCI* } \\
\text { (Lower, Upper) }\end{array}$} \\
\hline & & & & Noninferiority & Superiority & \\
\hline NDI ( $\geq 15$-point improvement) & $51 / 63(81.0 \%)$ & $111 / 125(88.8 \%)$ & 90/107 (84.1\%) & $100.0 \%$ & $92.1 \%$ & $(-2.5 \%, 15.7 \%)$ \\
\hline No removals, etc. & $61 / 65(93.8 \%)$ & $124 / 131(94.7 \%)$ & $102 / 121(84.3 \%)$ & $100.0 \%$ & $99.6 \%$ & $(2.4 \%, 16.9 \%)$ \\
\hline Neuro success & $59 / 63(93.7 \%)$ & $116 / 124(93.5 \%)$ & $92 / 105(87.6 \%)$ & $100.0 \%$ & $93.6 \%$ & $(-1.7 \%, 14.2 \%)$ \\
\hline No device-related adverse events & $61 / 65(93.8 \%)$ & $124 / 131(94.7 \%)$ & $100 / 121(82.6 \%)$ & $100.0 \%$ & $99.9 \%$ & $(3.8 \%, 18.6 \%)$ \\
\hline No change in treatment & $88 / 89(98.9 \%)$ & $148 / 151(98.0 \%)$ & $\mathrm{N} / \mathrm{A}$ & N/A & $\mathrm{N} / \mathrm{A}$ & $\mathrm{N} / \mathrm{A}$ \\
\hline Overall success & $50 / 65(76.9 \%)$ & $103 / 130(79.2 \%)$ & $77 / 121(63.6 \%)$ & $100.0 \%$ & $99.9 \%$ & $(5.7 \%, 27.0 \%)$ \\
\hline
\end{tabular}

Abbreviations: ACDF, anterior cervical discectomy and fusion; BCI, Bayesian credible intervals; NDI, neck disability index.

*BCI for difference in proportions (SECURE-C - ACDF).

proportion of patients classified as successes divided by the number of patients evaluated at the respective data collection time point.

According to the protocol-defined approach (Table 1), the overall success rate for the SECURE-C group was $86.3 \%$ as compared to $70.0 \%$ for the ACDF cohort at 84 months. For the FDAdefined approach (Table 2), the overall success rate for SECURE-C was $79.2 \%$ versus $63.6 \%$ for ACDF at 84 months. Statistical superiority of the investigational SECURE-C device over the control ACDF treatment at 84 months was established for both the protocol-specified and the FDA-defined overall success criteria, with a posterior probability of $99.9 \%$.

\section{NDI}

Preoperative NDI scores were not statistically different between the randomized SECURE-C $(51.8 \pm 13.84)$ and $\operatorname{ACDF}(51.5 \pm 14.86)$ groups. Both cohorts demonstrated improvement in NDI scores after surgery with similar trends during the study (Figure 1). Noninferiority of the randomized SECURE-C group was demonstrated at all postoperative visits for both $\geq 25 \%$ and $\geq 15$-point improvement success criteria. At 84 months, $90.4 \%$ of the randomized SECURE-C group demonstrated at least $25 \%$ improvement in NDI compared to $86.0 \%$ for the ACDF group, and

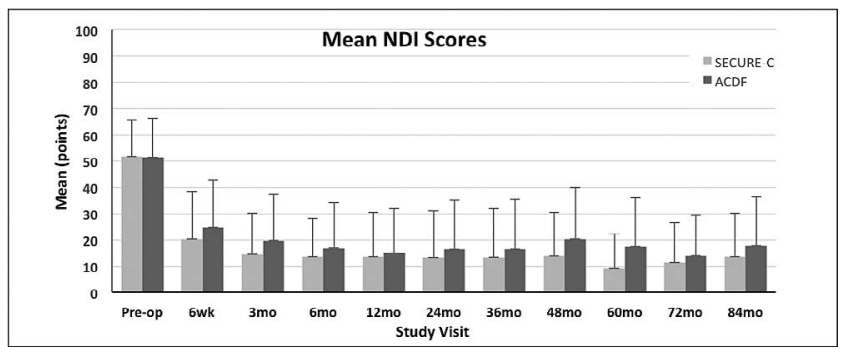

Figure 1. Mean neck disability index (NDI) scores (+SD) for the randomized SECURE-C and anterior cervical discectomy and fusion (ACDF) groups through 84 months postoperative.
$88.8 \%$ of randomized SECURE-C patients demonstrated at least a 15-point improvement in NDI compared to $84.1 \%$ of the ACDF patients.

\section{VAS Neck and Arm Pain}

VAS neck and arm pain scores were not statistically different between the randomized SECURE-C and ACDF groups at the preoperative baseline. Both cohorts demonstrated improvement in VAS neck and arm pain scores through 84 months postoperative (Figures 2 through 4 ). For VAS neck and arm pain improvement of at least $20 \mathrm{~mm}$ (or $0 \mathrm{~mm}$ postoperative pain), SECURE-C was statistically noninferior to ACDF. At 84 months, $85.7 \%$ of randomized SECURE-C patients demonstrated VAS neck pain success, compared to $78.3 \%$ of ACDF patients. For left and right arm pain at 84 months, $85.7 \%$ of randomized SECURE-C patients were VAS left-arm pain successes, compared to $75.5 \%$ of ACDF patients, and $84.9 \%$ of randomized SECURE-C patients were VAS right-arm pain successes, compared to $72.6 \%$ of ACDF patients. Preoperative and 84-month VAS pain scores along with success components are summarized in Tables 3 and 4 , respectively.

\section{Health Status Survey SF-36}

Both cohorts showed postoperative improvement in the SF-36 Physical Composite Score (PCS) and

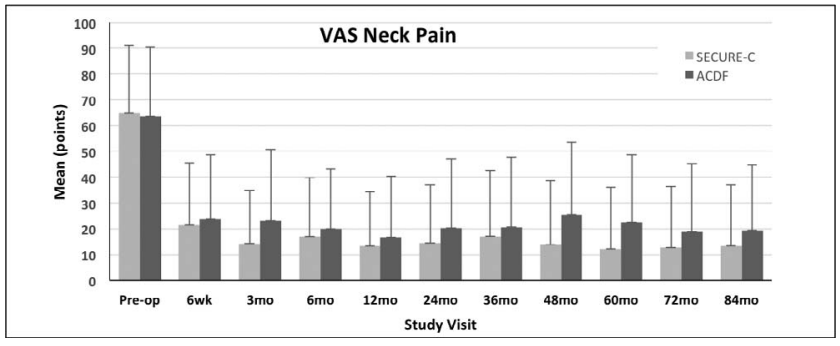

Figure 2. Mean visual analog scale (VAS) neck pain scores (+SD) for the randomized SECURE-C and anterior cervical discectomy and fusion (ACDF) groups through 84 months postoperative. 


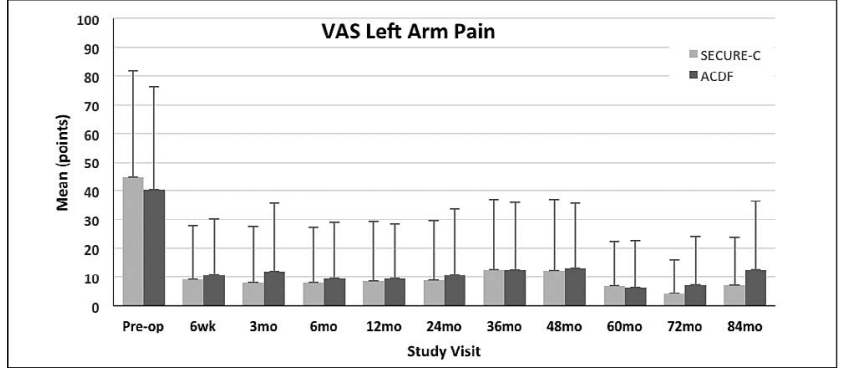

Figure 3. Mean visual analog scale (VAS) left arm pain scores (+SD) for the randomized SECURE-C and anterior cervical discectomy and fusion (ACDF) groups through 84 months postoperative.

Mental Composite Score (MCS) from preoperative through 84 months postoperative (Figure 5). Overall, there were no observable differences in SF-36 scores between the randomized SECURE-C group and the ACDF group. Preoperative and 84-month health status scores along with success components are summarized in Tables 3 and 4, respectively.

\section{Neurological Status}

Deterioration of any one of the 4 neurological assessments (motor, sensory, reflexes, other) from baseline preoperative values was considered deterioration of overall neurological status. Although not statistically significant, the randomized SECURE-C $(114 / 121 ; 94.2 \%)$ group experienced a higher percentage of patients with stable/improved neurological status than the control ACDF (94/108; $87.1 \%$ ) group at 84 months.

\section{Patient Satisfaction}

Ninety-six percent $(120 / 125)$ of patients treated in the randomized SECURE-C group responded that they were "definitely" or "mostly satisfied" with their surgery results; this rate was statistically superior to satisfaction reported among the ACDF group $(95 / 107 ; 88.8 \%)$.

\section{Adverse Events}

Adverse event rates were based on the number of patients having at least 1 occurrence of an adverse

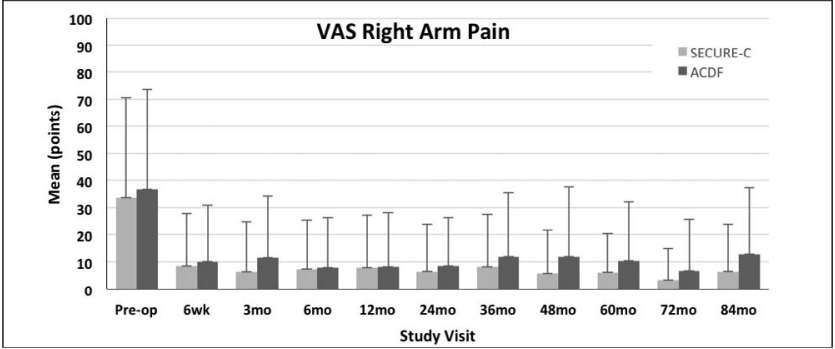

Figure 4. Mean visual analog scale (VAS) right arm pain scores $(+S D)$ for the randomized SECURE-C and anterior cervical discectomy and fusion (ACDF) groups through 84 months postoperative.

event divided by the number of patients in that treatment group. Device-related events consisted of neck pain, neck and upper extremity pain, and surgery at the index level. Adverse event rates and events per patient for both groups are summarized in Table 5. At 84 months postoperative, devicerelated (SECURE-C: $4.2 \%$ vs. ACDF: $15.3 \%$ ) and surgery-related (SECURE-C: $7.6 \%$ vs. ACDF: $15.3 \%$ ) adverse events were lower in the SECURE-C group than the ACDF group. No major differences were observed between SECURE-C and ACDF groups in terms of other adverse events, including severe or life-threatening complications.

\section{Secondary Surgical Procedures}

Some adverse events resulted in surgical intervention at the index level, subsequent to initial surgery. Secondary surgical interventions, classified as revisions, removals, reoperations, or supplemental fixation at the index level, were considered to be study failures. By at least 7 years postoperative, there were 10 secondary surgeries on patients treated with SECURE-C, whereas 22 secondary surgeries were performed on patients treated with ACDF. Out of the 10 surgeries in the SECURE-C patients, there were 6 removals, and the remaining 4 required posterior decompression without removal of the device. In the ACDF patients, 16 out of 22 secondary surgeries involved adjacent-level treat-

Table 3. Visual analog scale (VAS) and 36-Item Short Form Health Survey (SF-36) scores (mean \pm SD) at preoperative and 84 months follow-up.

\begin{tabular}{|c|c|c|c|c|}
\hline & \multicolumn{2}{|c|}{ SECURE-C } & \multicolumn{2}{|c|}{ ACDF } \\
\hline & Preoperative & $84 \mathrm{mo}$ & Preoperative & $84 \mathrm{mo}$ \\
\hline VAS neck pain & $64.7 \pm 26.37$ & $13.3 \pm 23.62$ & $63.5 \pm 26.81$ & $19.4 \pm 25.16$ \\
\hline VAS left arm pain & $44.8 \pm 37.05$ & $7.1 \pm 16.75$ & $40.3 \pm 36.08$ & $12.6 \pm 23.98$ \\
\hline VAS right arm pain & $33.8 \pm 36.87$ & $6.6 \pm 17.54$ & $36.8 \pm 36.99$ & $12.9 \pm 24.37$ \\
\hline SF-36 MCS & $44.0 \pm 13.16$ & $52.1 \pm 10.47$ & $44.4 \pm 11.97$ & $51.0 \pm 11.35$ \\
\hline $\mathrm{SF}-36 \mathrm{PCS}$ & $33.9 \pm 7.41$ & $46.4 \pm 12.07$ & $32.0 \pm 6.48$ & $44.7 \pm 10.90$ \\
\hline
\end{tabular}

Abbreviations: ACDF, anterior cervical discectomy and fusion; MCS, Mental Composite Score; PCS, Physical Composite Score. 
Table 4. Secondary component success summary at 84 months follow-up.*

\begin{tabular}{lccc}
\hline Component Success & SECURE-C $(\mathbf{N}=\mathbf{1 5 1})$ & ACDF $(\mathbf{N}=\mathbf{1 4 0})$ & $\mathbf{9 5 \%}$ BCI (Lower, Upper) \\
\hline NDI ( $\geq 25 \%$ improvement) & $113 / 125(90.4 \%)$ & $92 / 107(86.0 \%)$ & $(-3.6 \%, 13.3 \%)$ \\
NDI $(\geq 15$-point improvement) & $111 / 125(88.8 \%)$ & $90 / 107(84.1 \%)$ & $(-2.5 \%, 15.7 \%)$ \\
VAS neck pain & $108 / 126(85.7 \%)$ & $83 / 106(78.3 \%)$ & $(-2.7 \%, 17.5 \%)$ \\
VAS left arm pain & $108 / 126(85.7 \%)$ & $80 / 106(75.5 \%)$ & $(1.2 \%, 21.6 \%)$ \\
VAS right arm pain & $107 / 126(84.9 \%)$ & $77 / 106(72.6 \%)$ & $(0.7 \%, 21.0 \%)$ \\
SF-36 PCS & $90 / 125(72.0 \%)$ & $79 / 106(74.5 \%)$ & $(-14.3 \%, 8.0 \%)$ \\
SF-36 MCS & $59 / 125(47.2 \%)$ & $46 / 106(43.4 \%)$ & $(-9.6 \%, 14.5 \%)$ \\
\hline
\end{tabular}

Abbreviations: ACDF, anterior cervical discectomy and fusion; BCI, Bayesian credible intervals; NDI, neck disability index; VAS, visual analog scale; SF-36, 36-Item Short Form Health Survey; PCS, Physical Composite Score; MCS, Mental Composite Score.

*VAS pain score success ( $\geq 20$-mm improvement from baseline or 0-mm postoperative score). SF-36 health status PCS success ( $\geq 15 \%$ improvement) by visit. SF-36 health status MCS success ( $\geq 15 \%$ improvement) by visit.

ments. Moreover, the rate of secondary surgeries in the ACDF group was higher than the SECURE-C group throughout the study duration (Figure 6). The percentage of patients requiring secondary surgery was numerically lower for the SECURE-C group (4.2\%) than for the ACDF group (15.3\%).

\section{Radiological Assessments}

Anterior-posterior, lateral, and flexion-extension films obtained at predefined time points were utilized for radiographic assessments during the study. Range of motion success for the SECURE-C group was defined as at least $4^{\circ}$ of motion in flexionextension, or maintenance of motion relative to preoperative baseline. At 84 months, $85.1 \%$ of astreated (randomized + nonrandomized) SECURE-C patients were radiographic ROM successes (Figure 7). Overall, mean ROM in flexion-extension (Figure 8) and sagittal translation (Figure 9) were preserved and maintained with respect to preoperative values in the SECURE-C group at all follow-up time points during the study. Mean flexion-extension ROM for patients treated with SECURE-C was $9.2^{\circ}$ at 84 months, and mean sagittal translation was 1.1 $\mathrm{mm}$. For the control group, radiographic fusion was defined by the presence of bridging trabecular bone without evidence of pseudarthrosis and flexionextension ROM of $2^{\circ}$ or less in rotation and $3 \mathrm{~mm}$ or less in translation. All available 24-, 60-, and 84month radiographs for the SECURE-C treatment group were assessed for the presence of $\mathrm{HO}$ by an independent radiographic evaluator (Medical Metrics, Houston, Texas) using the Mehren classification system. ${ }^{7}$ At 84 months, 7.7\% (14/182) patients in the SECURE-C group were identified with evidence of Grade IV (complete fusion of the treated segment without movement) HO. Radiographic fusion was observed in 97.2\% (104/107) of patients who underwent ACDF at 84 months. Additionally, no radiolucencies were observed in either the SECURE-C or the ACDF cohorts at 84 months postoperative. There were no device breakages, migrations, or displacements, including superior-inferior subsidence reported among patients treated with SECURE-C during the course of the study.

\section{Adjacent Segment Treatment}

The incidence and progression of adjacent-level disease can be assessed in terms of symptomology, treatment, and surgery performed at an adjacent level. An in-depth analysis was performed on the entire SECURE-C study population to identify adverse events, treatments, and surgical interventions associated with adjacent-level symptoms.

Patients who experienced any postoperative symptoms potentially related to an adjacent level, such as radicular symptoms (arm pain or dysesthesia), neck pain, myelopathy, or any combination thereof, were thoroughly evaluated.

About sixty-two percent $(62.3 \% ; 147 / 236)$ of SECURE-C patients reported symptoms or treat-

Table 5. Adverse events by treatment group by at least 84 months of follow-up.

\begin{tabular}{lcrrr}
\hline & \multicolumn{2}{c}{ SECURE-C (N = 236) } & & \multicolumn{2}{c}{ ACDF (N = 144) } \\
\cline { 2 - 4 } Adverse Event (AE) & Patients (\%) & Events (E/Pt) & & Patients (\%) \\
\hline Any AE & $205(86.9 \%)$ & $723(3.06)$ & $130(90.3 \%)$ & Events (E/Pt) \\
Device-related AE & $10(4.2 \%)$ & $10(0.04)$ & $22(15.3 \%)$ & $537(3.73)$ \\
Surgery-related AE & $18(7.6 \%)$ & $19(0.08)$ & $22(15.3 \%)$ & $24(0.17)$ \\
Severe or life-threatening event & $87(36.9 \%)$ & $152(0.64)$ & $65(45.1 \%)$ & $105(0.73)$ \\
\hline
\end{tabular}

Abbreviation: $\mathrm{E} / \mathrm{Pt}$, events per patient. 


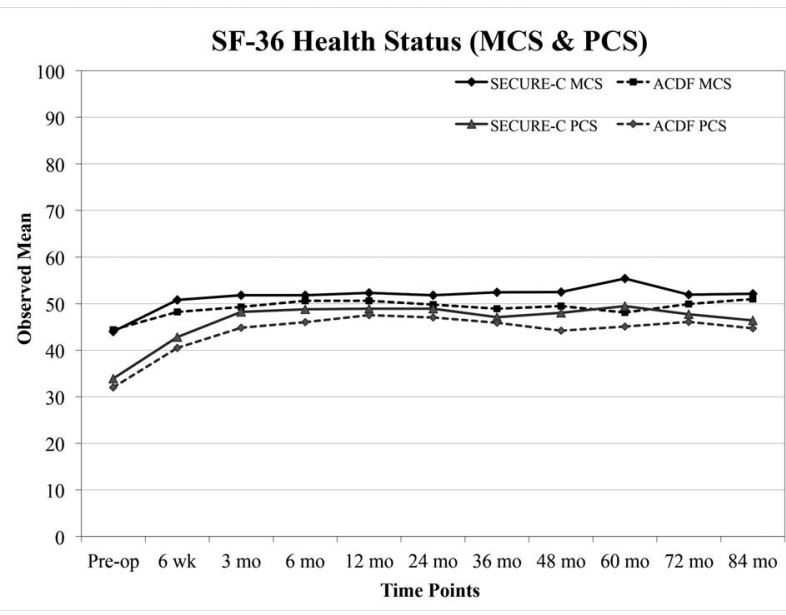

Figure 5. Mean 36-Item Short Form Health Survey (Mental Composite Score [MCS] and Physical Composite Score [PCS]) scores for the randomized SECURE-C and anterior cervical discectomy and fusion (ACDF) groups through 84 months postoperative.

ments possibly related to an adjacent level, whereas $72.9 \%(105 / 144)$ of ACDF patients had adverse events or interventions that could correlate to an adjacent level. On further analysis, a total of $17 \%$ (40/236) of SECURE-C patients experienced symptoms attributable to an adjacent level(s) in comparison to $37.5 \%(54 / 144)$ of ACDF patients.

A few patients with adjacent-level symptoms underwent nonsurgical treatments, such as epidural steroid injections, facet injections, and radio-frequency lesioning. Furthermore, a few patients with adjacent-level symptoms (with or without prior nonsurgical adjacent-level treatment) went on to receive postoperative surgical intervention at the adjacent level. ACDF patients had adjacent-level surgery earlier and more often than SECURE-C patients. The percentage of patients with adjacentlevel surgery was low for both groups, with 5 or fewer patients receiving treatment within each postoperative window. However, the rate of patients receiving adjacent-level surgery increased in the ACDF group and was higher than the SECURE-C group during the course of the study (Figure 10). Cumulatively, at 84 months postoperative, the percentage of ACDF $(16 \% ; 23 / 144)$ patients undergoing adjacent-level surgical treatment was higher than the percentage of SECURE-C $(4.2 \% ; 10 / 236)$ patients.

\section{DISCUSSION}

Cervical arthroplasty devices are designed to provide relief of clinical problems attributed to symptomatic CDD, similar to traditional fusion

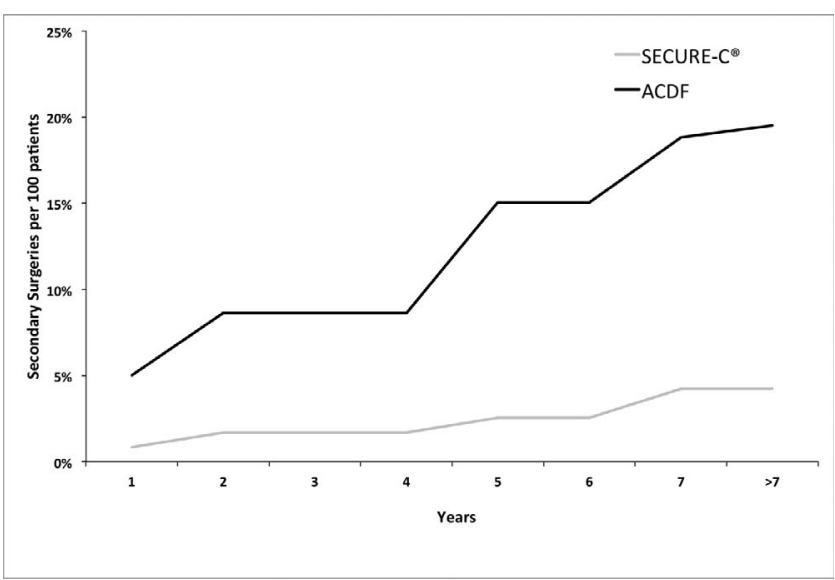

Figure 6. Cumulative secondary surgeries at treated level by at least 84 months postoperative. Abbreviation: ACDF, anterior cervical discectomy and fusion.

procedures. However, unlike fusion, which restricts motion, arthroplasty preserves motion and may act as a preventive modality to mitigate or delay the onset of degeneration of adjacent segments, thereby decreasing the probability of subsequent interventions. Although cervical arthroplasty devices have been available for more than 3 decades, the adoption of the technology has been limited partly due to uncertainty about long-term clinical outcomes associated with these devices as well as the perceived clinical success of traditional cervical fusion surgeries. The clinical IDE results presented here are consistent with other recent reports demonstrating the long-term safety and efficacy of cervical arthroplasty and its potential benefits in comparison to traditional cervical fusion. ${ }^{8-19}$

Overall success in the IDE protocol was defined as a composite score of parameters that are necessary to reasonably evaluate the primary effectiveness of an investigational group in comparison to the control group. A patient is considered an individual success if and only if all of the parameters are considered a success, rendering it a rigorous scoring metric. Furthermore, an additional FDArequested overall success criterion was implemented that required a more conservative approach in defining parameters, essentially decreasing the potential for measurement bias between the groups. Statistical superiority of the SECURE-C group over ACDF was achieved according to the stricter FDAdefined criteria as well as the protocol-defined criteria at the 24- and 84-month time points, with similar trends during intermediate follow-up periods. Although no statistical difference was observed in terms of NDI success and neurological success, 

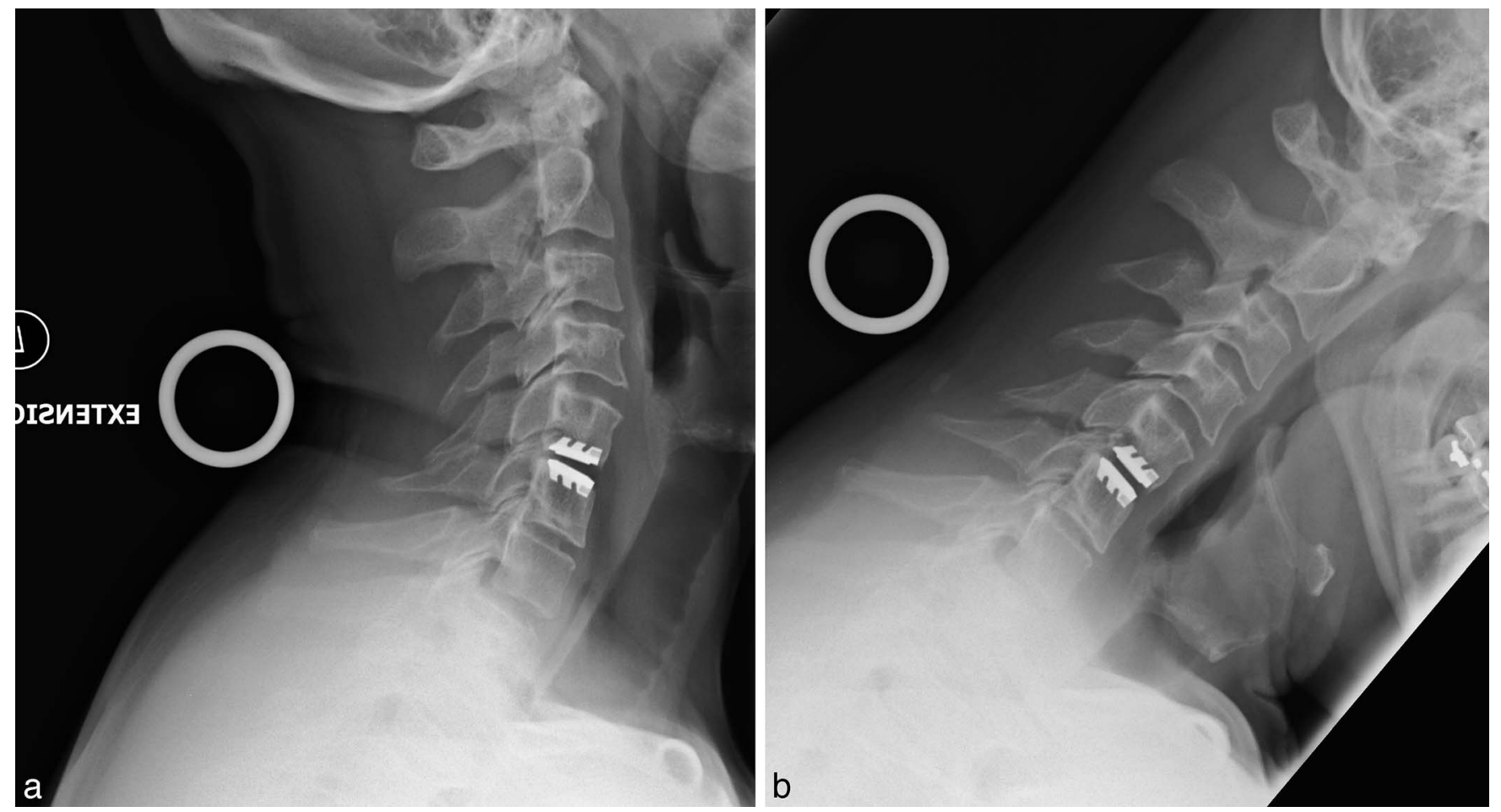

Figure 7. Postoperative radiographs obtained at 84 months showing SECURE-C device in (a) flexion and (b) extension.

fewer device-related adverse events and removals in the SECURE-C group were instrumental for a greater overall success composite score in relation to the ACDF group. At 7 years postoperative, $86.3 \%$ of patients in the SECURE-C cohort satisfied the protocol-defined criteria for overall success, which is numerically greater than what was observed for similar cervical arthroplasty devices such as Prestige LP (7 year: $74.9 \%)^{13}$ and Mobi-C (5 year: $\left.61.9 \%\right){ }^{14}$ Although the differences in these composite scores are attributable to multiple factors other than device characteristics, these findings render evidentiary

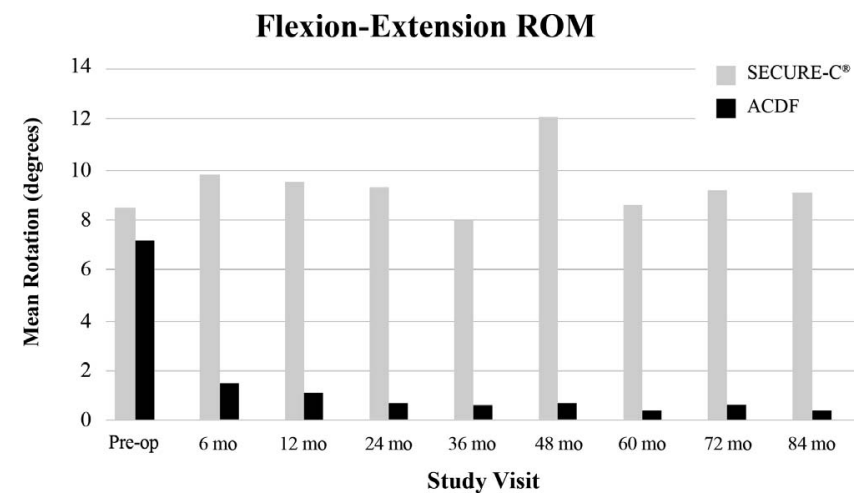

Figure 8. Mean flexion-extension range of motion (ROM) at the operated level through 84 months postoperative. Abbreviation: ACDF, anterior cervical discectomy and fusion. support for favorable long-term prognoses with cervical arthroplasty in general.

Functional improvement and overall well-being were documented using patient-reportable outcome measures such as NDI, SF-36, and VAS neck and arm pain, along with a patient satisfaction questionnaire. These are commonly used tools for evaluating the treatment effectiveness of cervical spine surgery. Both groups experienced marked improvements in pain scores, strongly suggesting that the majority of symptom relief was provided by neural decompression, as this is a common denominator in both fusion and arthroplasty surgeries. The scale of improvement and maintenance of pain status in both groups indicate that both procedures

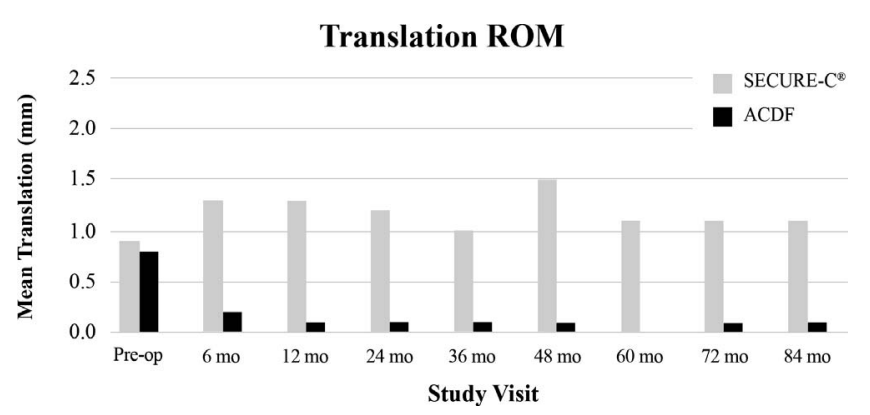

Figure 9. Mean translation range of motion (ROM) at the operated level through 84 months postoperative. Abbreviation: ACDF, anterior cervical discectomy and fusion. 


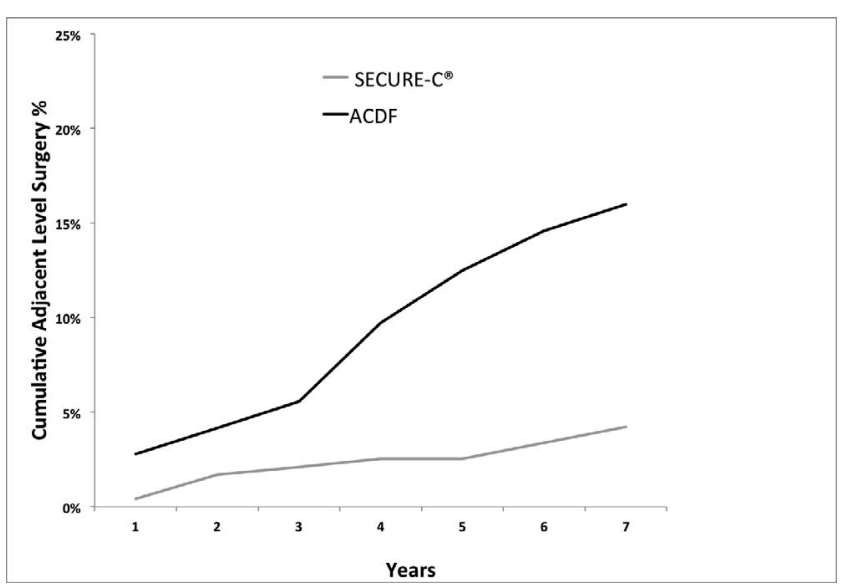

Figure 10. Cumulative adjacent level surgeries through 84 months postoperative. Abbreviation: ACDF, anterior cervical discectomy and fusion.

provided at the least minimum clinically important difference, if not the ultimate goal of a significant clinical benefit, as defined by the clinical community. ${ }^{20}$

Adverse events and secondary surgeries are impactful clinical factors that result in significant medical and financial burden to the patient and the payer. Contemporary studies have suggested that total disc arthroplasty can be cost-effective, primarily by decreasing the rate of secondary surgeries. $^{21-25}$ In this study, device- and surgery-related adverse events were lower in the SECURE-C group than in the ACDF group. In addition, the rate of secondary surgery at the treated index level was lower in the SECURE-C group than fusion, supporting trends reported in other studies comparing arthroplasty to ACDF. ${ }^{26-28}$

The majority of SECURE-C patients showed an increase in and maintenance of ROM after surgery. At 84 months postoperative, $85.1 \%$ of SECURE-C patients were considered ROM successes based on study criteria of exhibiting $\geq 4^{\circ}$ of flexion-extension ROM. A small percentage of patients (7.7\%) showed radiographic evidence of $\mathrm{HO}$ to the extent of limiting motion at the treated level, which is consistent with other studies on cervical arthroplasty. ${ }^{11-19}$ While the etiology of $\mathrm{HO}$ and its effect on motion preservation is an important consideration, its association with either clinical relevance ${ }^{29-32}$ or patient outcome $\mathrm{s}^{33,34}$ after arthroplasty remains unclear. It is suggested that reducing bleeding bone, maximizing endplate coverage ${ }^{35}$ with the arthroplasty device, and nonsteroidal anti-inflammatory drug prophylaxis may be beneficial in reducing the occurrence of $\mathrm{HO}$.
One of the primary advantages of arthroplasty is the potential to mitigate or delay the symptoms of adjacent segment disease. ${ }^{5,36-38}$ In this study, longterm follow-up demonstrated that patients treated with SECURE-C had fewer adjacent segment surgeries when compared to the fusion group, suggesting the protective role of arthroplasty on adjacent segments. These findings are consistent with other reports indicating a protective role of motion preservation with respect to the occurrence of adjacent-level symptoms. ${ }^{13,15,18}$ However, the decision-making process in electing treatment for adjacent segment surgery is not well defined and may be subject to surgeon bias. Moreover, the state of degeneration of adjacent segments, prior to implantation, may influence the rate of adjacent segment breakdown.

There are limitations to this study. The randomized controlled clinical trial was designed with strict inclusion and exclusion criteria. Outcomes of patients in the real world may differ. The study compared the safety and efficacy of the SECURE-C Cervical Artificial Disc against fusion, and the results cannot be generalized to other cervical arthroplasty devices. Despite enrolling a nonrandomized group of 5 SECURE-C patients per investigational site for initial training purposes, the limited population of the clinical study would still be considered an early learning curve for each investigator surgeon. It is reasonable to predict that additional surgical experience with appropriate patient selection may lead to improved outcomes.

\section{CONCLUSION}

Seven-year follow-up of 380 patients enrolled in the prospective, randomized, controlled, clinical IDE trial demonstrated that SECURE-C is noninferior to $\mathrm{ACDF}$ in terms of providing long-term pain relief and functional improvement in patients diagnosed with single-level CDD refractory to nonoperative treatment. Study results demonstrated that the SECURE-C cohort was statistically superior to ACDF in terms of composite overall success and patient satisfaction. SECURE-C patients also experienced lower rates of subsequent index- and adjacent-level surgical interventions. Given the level 1 data presented herein and in consideration of the long-term results of prospective clinical trials for other cervical artificial discs, it is prudent to reevaluate categorization of treatment options for symptomatic CDD. 


\section{ACKNOWLEDGMENTS}

The authors would like to acknowledge the following surgeons and their staffs for their role as participants/contributors in the SECURE-C IDE trial: Joseph M. Marzluff, MD, Jason Highsmith, MD, Jeffrey McConnell, MD, James Weis, MD, Christopher Tomaras, MD, Thomas Morrison, MD, Max Steuer, MD, Kevin Stevenson, MD, John Small, MD, James Billys, MD, James Lindley, MD, Robert Robinson, MD, Scott Rushton, MD, Ira Fedder, MD, Paul McAfee, MD, Walter Peppelman, DO, William Beutler, MD, Steven Wolf, MD, Jeffrey Cattorini, MD, John Tompkins, MD, Rebecca Stachniak, MD, Leslie Hellbusch, MD, Bradley Bowdino, MD, Douglas Long, MD, Mark Palumbo, MD, Frederick Harrington, MD, Prakash Sampath, MD, Hoang Le, MD, George Shanno, MD, Norman Rokosz, MD, Paul Asdourian, MD, Shane Pak, MD, Michael Kaiser, MD, David Goss, MD, Michael Barnum, MD, Ildemaro Volcan, MD, Allan Goodrich, MD, Larry Parker, MD, and Morris Seymour, MD.

\section{REFERENCES}

1. Davis H. Increasing rates of cervical and lumbar spine surgery in the United States, 1979-1990. Spine (Phila Pa 1976). 1994;19(10):1117-1124.

2. Rajaee SS, Bae HW, Kanim LE, Delamarter RB. Spinal fusion in the United States: analysis of trends from 1998 to 2008. Spine (Phila Pa 1976). 2012;37(1):67-76.

3. Lad SP, Patil CG, Berta S, Santarelli JG, Ho C, Boakye M. National trends in spinal fusion for cervical spondylotic myelopathy. Surg Neurol. 2009;71(1):66-69.

4. Durbhakula MM, Ghiselli G. Cervical total disc replacement, part I: rationale, biomechanics, and implant types. Orthop Clin N Am. 2005; 36(3):349-354.

5. Hilibrand AS, Carlson GD, Palumbo MA, et al. Radiculopathy and myelopathy at segments adjacent to the site of a previous anterior cervical arthrodesis. $J$ Bone Joint Surg Am. 1999; 81(4):519-528.

6. Vaccaro A, Beutler W, Peppelman W, et al. Clinical outcomes with selectively constrained SECURE-C cervical disc arthroplasty: two-year results from a prospective, randomized, controlled, multicenter investigational device exemption study. Spine (Phila Pa 1976). 2013;38(26):2227-2239.

7. Mehren C, Suchomel P, Grochulla F, et al. Heterotopic ossification in total cervical artificial disc replacement. Spine (Phila Pa 1976). 2006;31(24):2802-2806.

8. Heller JG, Sasso RC, Papadopoulos SM, et al. Comparison of BRYAN cervical disc arthroplasty with anterior cervical decompression and fusion: clinical and radiographic results of a randomized, controlled, clinical trial. Spine (Phila Pa 1976). 2009;34(2):101-107.

9. Coric D, Nunley PD, Guyer RD, et al. Prospective, randomized, multicenter study of cervical arthroplasty: 269 patients from the Kineflex $\mid \mathrm{C}$ artificial disc investigational device exemption study with a minimum 2-year follow-up: clinical article. J Neurosurg Spine. 2011;15(4):348-358.

10. Kim SH, Shin HC, Shin DH. Early clinical experience with the mobi-C disc prosthesis. Yonsei Med J. 2007;48(3):457464.

11. Mummaneni PV, Burkus JK, Haid RW, et al. Clinical and radiographic analysis of cervical disc arthroplasty compared with allograft fusion: a randomized controlled clinical trial. J Neurosurg Spine. 2007;6(3):198-209.

12. Murrey D, Janssen M, Delamarter R. Results of the prospective, randomized, controlled multicenter Food and Drug Administration investigational device exemption study of the ProDisc-C total disc replacement versus anterior discectomy and fusion for the treatment of 1-level symptomatic cervical disc disease. Spine J. 2009;9(4):275-286.

13. Gornet MF, Burkus JK, Shaffrey ME, Nian H, Harrell FE Jr. Cervical disc arthroplasty with prestige LP disc versus anterior cervical discectomy and fusion: seven-year outcomes. Int J Spine Surg. 2016;10:24.

14. Hisey MS, Zigler JE, Jackson R, et al. Prospective, randomized comparison of one-level Mobi-C cervical total disc replacement vs. anterior cervical discectomy and fusion: results at 5-year follow-up. Int J Spine Surg. 2016;10:10.

15. Hisey MS, Bae HW, Davis RJ, et al. Prospective, randomized comparison of cervical total disk replacement versus anterior cervical fusion: results at 48 months follow-up. $J$ Spinal Disord Tech. 2015;28(4):E237-E243.

16. Zigler JE, Delamarter R, Murrey D, et al. ProDisc-C and anterior cervical discectomy and fusion as surgical treatment for single-level cervical symptomatic degenerative disc disease: five-year results of a Food and Drug Administration study. Spine (Phila Pa 1976). 2013;38(3):203-209.

17. Burkus JK, Haid RW, Traynelis VC, et al. Long-term clinical and radiographic outcomes of cervical disc replacement with the Prestige disc: results from a prospective randomized controlled clinical trial. J Neurosurg Spine. 2010;13(3):308-318.

18. Burkus JK, Traynelis VC, Haid RW Jr, et al. Clinical and radiographic analysis of an artificial cervical disc: 7-year follow-up from the Prestige prospective randomized controlled clinical trial: clinical article. J Neurosurg Spine. 2014;21(4):516528.

19. Quan GM, Vital JM, Hansen S, et al. Eight-year clinical and radiological follow-up of the Bryan cervical disc arthroplasty. Spine (Phila Pa 1976). 2011;36(8):639-646.

20. Katz NP, Paillard FC, Ekman E. Determining the clinical importance of treatment benefits for interventions for painful orthopedic conditions. J Orthop Surg Res. 2015;10:24.

21. Radcliff K, Zigler J, Zigler J. Costs of cervical disc replacement versus anterior cervical discectomy and fusion for treatment of single-level cervical disc disease: an analysis of the Blue Health Intelligence database for acute and long-term costs and complications. Spine (Phila Pa 1976). 2015;40(8):521-529.

22. McAnany SJ, Overley S, Baird EO, et al. The 5-year cost-effectiveness of anterior cervical discectomy and fusion and cervical disc replacement: a Markov analysis. Spine (Phila Pa 1976). 2014;39(23):1924-1933.

23. Warren D, Andres T, Hoelscher C, Ricart-Hoffiz P, Bendo J, Goldstein J. Cost-utility analysis modeling at 2-year 
follow-up for cervical disc arthroplasty versus anterior cervical discectomy and fusion: a single-center contribution to the randomized controlled trial. Int J Spine Surg. 2013;7:e58-e66.

24. Qureshi SA, McAnany S, Goz V, et al. Costeffectiveness analysis: comparing single-level cervical disc replacement and single-level anterior cervical discectomy and fusion. J Neurosurg Spine. 2013;19(5):546-554.

25. Ament J. Cost utility analysis of the cervical artificial disc vs fusion for the treatment of 2-level symptomatic degenerative disc disease: 5-year follow-up. Neurosurgery. 2016;79(1):135-145.

26. Blumenthal SL, Ohnmeiss DD, Guyer RD, et al. Reoperations in cervical total disc replacement compared with anterior cervical fusion: results compiled from multiple prospective FDA IDE trials conducted at a single site. Spine (Phila Pa 1976). 2013;38(14):1177-1182.

27. Delamarter RB, Zigler J. Five-year reoperation rates, cervical total disc replacement versus fusion, results of a prospective randomized clinical trial. Spine (Phila Pa 1976). 2013;38(9):711-717.

28. Jackson RJ, Davis RJ, Hoffman GA, et al. Subsequent surgery rates after cervical total disc replacement using a Mobi-C Cervical Disc Prosthesis versus anterior cervical discectomy and fusion: a prospective randomized clinical trial with 5-year follow-up. J Neurosurg Spine. 2016;24(5):734-745.

29. Leung C, Casey AT, Goffin J, et al. Clinical significance of heterotopic ossification in cervical disc replacement: a prospective multicenter clinical trial. Neurosurgery. 2005;57(4):759-763.

30. Cho YH, Kim KS, Kwon YM. Heterotopic ossification after cervical arthroplasty with ProDisc-C: time course radiographic follow-up over 3 years. Korean $J$ Spine. 2013;10(1):19-24.

31. Guerin P, Obeid I, Bourghli A, et al. Heterotopic ossification after cervical disc replacement: Clinical significance and radiographic analysis: a prospective study. Acta Orthop Belg. 2012;78(1):80-86.

32. Walraevens J, Demaerel P, Suetens P, et al. Longitudinal prospective long-term radiographic follow-up after treatment of single-level cervical disk disease with the Bryan cervical disc. Neurosurgery. 2010;67(3):679-687.

33. Rihn JA, Radcliff K, Hipp J, et al. Radiographic variables that may predict clinical outcomes in cervical disc replacement surgery. J Spinal Disord Tech. 2015;28(3):106-113.

34. Chen J, Wang X, Bai W, Shen X, Yuan W. Prevalence of heterotopic ossification after cervical total disc arthroplasty: a meta-analysis. Eur Spine J. 2012;21(4):674-680.

35. Nunley P, Cavanaugh D, Ker EJ, et al. Heterotopic ossification and endplate sizing in one-level CDA patients: patient outcomes from a randomized, prospective, clinical trial. Proceedings from International Society for the Advancement of Spine Surgery; April 15-17, 2015; San Diego, CA.

36. Jawahar A, Cavanaugh DA, Kerr EJ III, Birdsong EM, Nunley PD. Total disc arthroplasty does not affect the incidence of adjacent segment degeneration in cervical spine: results of 93 patients in three prospective randomized clinical trials. Spine J. 2010;10(12):1043-1048

37. Nunley PD, Jawahar A, Kerr EJ III, et al. Factors affecting the incidence of symptomatic adjacent level disease in cervical spine after total disc arthroplasty: 2- to 4-year followup of 3 prospective randomized trials. Spine (Phila Pa 1976). 2012;37(6):445-451.

38. Luo J, Gong M, Huang S, Yu T, Zou X. Incidence of adjacent segment degeneration in cervical disc arthroplasty versus anterior cervical decompression and fusion meta-analysis of prospective studies. Arch Orthop Trauma Surg. 2015;135(2):155-160.

Corresponding Author: Prem S. Ramakrishnan, PhD, Globus Medical, 2560 General Armistead Avenue, Audubon, PA 19403, Phone: (610)930-1800; Email: pramakrishnan@ globusmedical.com.

Published 15 August 2018

This manuscript is generously published free of charge by ISASS, the International Society for the Advancement of Spine Surgery. Copyright $\odot 2018$ ISASS. To see more or order reprints or permissions, see http://ijssurgery.com. 